\title{
Isolation drives reward-seeking in rats
}

\author{
Social isolation can lead to poor mental and physical health. A new study determines that social isolation increases \\ food and nicotine-seeking during abstinence, but that social housing can reverse these effects.
}

\section{Rebecca S. Hofford}

S ocial isolation is increasing in the days of the COVID-19 crisis. While social distancing and business and school closures are necessary to help curb the spread of disease, the resulting isolation and loneliness contributes to adverse health consequences including increased incidences of depression, anxiety, and substance abuse. Understanding how social isolation affects the consumption and seeking of both natural rewards and drugs of abuse is important - this knowledge can help identify at-risk individuals and can help individualize psychosocial therapies. A recent paper by Natalie Mastrogiovanni et al. ${ }^{1}$ examines the effects of social isolation on food and nicotine taking and reinstatement, finding that socially isolating rats increases food and nicotine seeking when these rewards are not available.

The major goal of the current paper involved measuring food and nicotine-seeking after social isolation by utilizing probe sessions during self-administration maintenance (i.e. an active period of drug intake after behavior has stabilized) and reinstatement after extinction (i.e. operant responding for cues after abstinence). First, animals living in social isolation or group housing learned to perform an action - in this study, poking their noses into a portal - to receive either a sucrose pellet or an infusion of nicotine as a reinforcer. During training, the reinforcer was delivered simultaneously with illumination of a cue light, which by itself would have little value but gains incentive salience due to its consistent occurrence with the reinforcer. After training, such cues can cause an action even in the absence of the reinforcer ${ }^{2}$, an important consideration for modeling addiction in animals. Enhanced motivational value placed on food- or drug-paired cues can be problematic, as compulsive eating or excessive drug use can be initiated by otherwise innocuous objects or symbols that are abundant in the environment. For example, it has been shown in binge eaters or individuals with a substance use disorder that encounters with food ${ }^{3}$

Experiment 1 (pellet) and 2 (nicotine)

\begin{tabular}{|c|c|c|c|c|c|c|c|}
\hline Grouped housed (GH) & 으 & & & & & & $\dot{s}$ \\
\hline 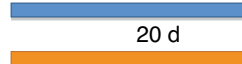 & 苞 & $\begin{array}{c}\text { Acquisition (FR-1) } \\
(12-16 \mathrm{~d})\end{array}$ & $\begin{array}{l}\text { Probe } \\
\text { test } \\
(4 \mathrm{~d})\end{array}$ & $\begin{array}{c}\text { Extinction } \\
(<4-6 \mathrm{~d})\end{array}$ & 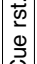 & $\mid$ & 㤐 \\
\hline Socially isolated (SI) & $\stackrel{\mathbb{\infty}}{I}$ & & & & & & 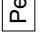 \\
\hline
\end{tabular}

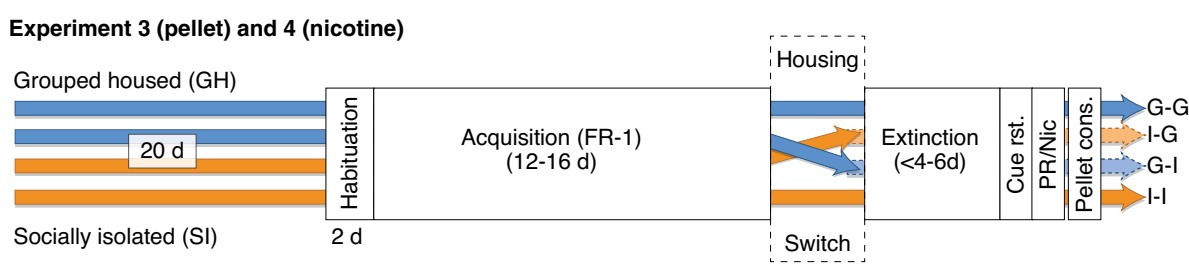

Fig. 1 | Mastrogiovanni et al.'s experimental design tested adult rats' pellet and nicotine consumption over time and after a change in environmental enrichment. Adapted from Mastrogiovanni et al (2021). Springer Nature!

or drug cues ${ }^{4}$ during remission are major drivers of relapse.

In the current study, probe sessions started the day after training and allowed rats to respond to either the cue light alone or the reinforcer alone. After behavior had been extinguished by repeated sessions where active responding no longer produced a cue light or a reinforcer, cue-induced reinstatement sessions allowed rats to nose poke for the cue alone. Both cue probe sessions and cue-induced reinstatement measure the ability of the cue to drive operant behavior; however, because extinction is necessary for reinstatement, performance on these tasks requires different brain circuits.

This paper builds upon the existing literature in several meaningful ways, including limiting the period of social isolation to adulthood and employing a within-subject housing switch to identify the experimental phase responsible for alterations in behavior (Fig. 1). Prior research has determined that the rearing environment of rodents can influence behavioral responses to natural reinforcers ${ }^{5,6}$ and drugs of abuse $\mathrm{e}^{7-12}$. Many studies have compared the behavior of rats raised in environmental enrichment to the behavior of rats raised in impoverished conditions.
Although social housing manipulations can differ by lab, environmental enrichment typically consists of a large housing arena containing physical objects that vary day to day as well as social conspecifics. On the opposite end of the enrichment spectrum is impoverishment, where rats are confined by themselves to a smaller space that often lacks bedding and physical objects. When utilizing these disparate environments, obvious differences in food-maintained responding and drug intake manifest, with impoverished animals self-administering more cocaine $\mathrm{e}^{7}$, amphetamine ${ }^{11}$, alcohol ${ }^{8}$, nicotine $^{10}$, methylphenidate ${ }^{12}$, and opioids ${ }^{9}$. In contrast to impoverishment, environmental enrichment protects against high levels of drug taking and reinstatement across a wide range of drug classes. Likewise, studies utilizing less extreme iterations of these housing conditions produce similar results (for example, see ${ }^{13,14}$ ), together suggesting that the less robust the physical and social housing environment, the greater the drug intake and the more drug-seeking behavior.

Despite the plethora of studies showing detrimental effects of social isolation on rates of drug taking in rodents, alterations of housing conditions often start immediately post-weaning or during 
adolescence and remain the same for the duration of the study. While examining the behavioral consequences of enrichment and impoverishment in adolescence is a worthwhile endeavor, social isolation is not limited to this time of life and can occur at any age. In this study ${ }^{1}$, the authors socially isolated adult rats to investigate the effects of isolation on older animals. Adult rats were divided into either group housing (GH) or social isolation (SI). Group housing consisted of four rats per cage within a large arena with moderate levels of enrichment while socially isolated rats were singly housed in a medium-sized arena with the same amount of enrichment as the GH rats. The authors found that SI sped acquisition of food-reinforced responding but had no effect on nicotine intake. However, differences emerged during nicotine probe sessions. GH rats showed significantly greater responding for the cue light compared to nicotine infusion, and they also had greater cue-induced reinstatement than $\mathrm{GH}$ rats undergoing nicotine probe sessions. SI rats meanwhile responded equally for cue and nicotine during probe sessions but SI rats experiencing the nicotine probe demonstrated greater levels of cue-induced reinstatement.

It is interesting that $\mathrm{GH}$ rats responded more for a nicotine-paired cue than nicotine itself while SI rats responded equally for cue and nicotine during probe sessions. As mentioned in the discussion of the original paper, this could suggest that $\mathrm{GH}$ rats form a stronger association between cue and reinforcer or learn this relationship more quickly than SI rats. This could be in line with the environmental enrichment literature that showed that environmentally enriched rats have a greater nicotine place preference than impoverished rats across a range of nicotine doses $^{15}$ despite less low-dose nicotine intake in self-administering rats raised in these environments ${ }^{10}$. Conditioned place preference (CPP) is entirely dependent upon learned associations between the interoceptive effects of drugs and the drug-paired environment, suggesting that cues associated with nicotine (cue light during self-administration or drug-paired environment during CPP) might gain more incentive value in rats living in a more robust housing condition. More work needs to be done to determine if this general effect carries over to other drugs of abuse and work needs to be done to determine the functional consequences of reduced incentive salience attribution in SI rats.

The second novel aspect of this paper was the switching of housing types before reinstatement. As stated previously, past literature has solidly established that housing in deprived environments can enhance drug intake ${ }^{8-11}$ and cue-induced reinstatement ${ }^{16,17}$ when housing is established prior to training. Additional literature suggests that starting social housing during abstinence from self-administration is also capable of reducing reinstatement ${ }^{18-20}$. While some studies have switched housing conditions during periods of active self-administration $^{10,21}$, it was unknown whether a switch in housing between self-administration and reinstatement would influence food or drug seeking after extinction. The experimental design used in the current study separates the effect of housing condition on the development of the reinforcer-cue association from the effect of housing on the expression of the seeking response. For instance, if a strong association has been established between the cue and the reinforcer during training, a switch in housing before extinction should have little effect on the seeking response. If, however, the housing environment at testing is most important for food or nicotine-seeking, housing condition during training should not influence reinstatement.

For reinstatement of food responding, housing condition during training had the greatest influence on food seeking. Rats previously housed in SI during acquisition (Isolated $\rightarrow$ Isolated, I-I, and Isolated $\rightarrow$ Group, I-G), had higher rates of responding than previously $\mathrm{GH}$ rats (Group $\rightarrow$ Group, G-G, and Group $\rightarrow$ Isolated, G-I). Interestingly, G-I rats had the lowest response rate while I-I rats had the highest, suggesting perhaps that the housing switch itself drove animals to have lower response rates regardless of whether the switch was from a more robust environment $(\mathrm{GH})$ to a less robust environment (SI) or vice versa. For cue-induced nicotine-seeking after housing condition switch, the results were also mixed. Housing condition during training and during reinstatement test clearly influenced the behavior of rats previously housed in SI. In contrast, GH rats during training (G-G and G-I) both had reduced levels of cue-induced reinstatement compared to I-I and I-G, but re-housing these rats had no effect on nicotine-seeking (G-G = G-I).

The results of Mastrogiovanni et al. raise some interesting questions and open new avenues of research. This study and past literature clearly indicate the robust effect of social environment on food and drug seeking. While understanding the extent and limitations of social housing as a potential psychosocial therapeutic is valuable in its own right, performing these studies in rodents also allows investigators to probe the potential neurobiological mechanism underlying the protective effects of social housing on food and drug-seeking. Future studies can identify pharmacological targets of interest to combine pharmaceutical approaches and psychosocial intervention to individualize combination treatments that help the most at-risk individuals.

\section{Rebecca S. Hofford (D) $₫$}

Icahn School of Medicine at Mount Sinai, 1470 Madison Ave, HCSM 10-302, New York, NY 10029, USA.

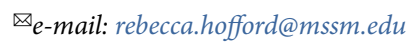

Published online: 29 March 2021

https://doi.org/10.1038/s41684-021-00756-5

References

1. Mastrogiovanni, N. A., Wheeler, A. K. \& Clemens, K. J. Sci. Rep. 11, 2422 (2021).

2. Berridge, K. C. \& Aldridge, J. W. Oxford Ser. Soc. Cogn. Soc. Neurosci. 2009, 509-533 (2009).

3. Jansen, A. Behav. Res. Ther. 36, 257-272 (1998).

4. McHugh, R. K., Fulciniti, F., Mashhoon, Y. \& Weiss, R. D. Am. J. Addict. 25, 105-109 (2016).

5. Gill, M. J. \& Cain, M. E. Behav. Pharmacol. 22, 40-48 (2011).

6. Cain, M. E., Green, T. A. \& Bardo, M. T. Behav. Processes 73, 360-366 (2006).

7. Green, T. A. et al. Biol. Psychiatry 67, 28-35 (2010).

8. Deehan, G. A. Jr., Cain, M. E. \& Kiefer, S. W. Alcohol. Clin. Exp. Res. 31, 1692-1698 (2007)

9. Hofford, R. S., Chow, J. J., Beckmann, J. S. \& Bardo, M. T. Psychopharmacology (Berl). (2017). https://doi.org/10.1007/ s00213-017-4734-2

10. Powell, G. L. et al. Drug Alcohol Depend. 201, 188-196 (2019).

11. Bardo, M. T., Klebaur, J. E., Valone, J. M. \& Deaton, C. Psychopharmacology (Berl). 155, 278-284 (2001).

12. Alvers, K. M., Marusich, J. A., Gipson, C. D., Beckmann, J. S. \& Bardo, M. T. Behav. Pharmacol. 23, 650-657 (2012).

13. Fosnocht, A. Q., Lucerne, K. E., Ellis, A. S., Olimpo, N. A. \& Briand, L. A. Behav. Brain Res. 359, 589-596 (2019).

14. Bozarth, M. A., Murray, A. \& Wise, R. A. Pharmacol. Biochem. Behav. 33, 903-907 (1989).

15. Ewin, S. E., Kangiser, M. M. \& Stairs, D. J. Experimental and Clinical Psychopharmacology 23, 387-394 (2015).

16. Arndt, D. L., Wukitsch, T. J., Garcia, E. J. \& Cain, M. Behav. Neurosci. 133, 478-488 (2019).

17. Hofford, R. S., Darna, M., Wilmouth, C. E., Dwoskin, L. P. \& Bardo, M. T. Behav. Brain Res. 270, (2014).

18. Gauthier, J. M. et al. Addict. Biol. 22, 152-162 (2017).

19. Powell, G. L. et al. Sci. Rep. 10, 11291 (2020).

20. Chauvet, C., Lardeux, V., Goldberg, S. R., Jaber, M. \& Solinas, M. Neuropsychopharmacology 34, 2767-2778 (2009).

21. Yates, J. R., Bardo, M. T. \& Beckmann, J. S. Addict. Biol. 24, 65-75 (2019). 